\title{
Determinants of perceived health care provider empathy at public and private hospitals in central Ethiopia
}

\author{
Tayue Tateke ${ }^{1, *}$, Mirkuzie Woldie ${ }^{2}$, Shimeles Ololo ${ }^{2}$ \\ ${ }^{1}$ Collegeof Medicine and Health Sciences, Arbaminch University, P.O.Box 80583, Addis Ababa, Ethiopia \\ ${ }^{2}$ Department of Health Services Management, Jimma University, Jimma, Ethiopia
}

\section{Email address:}

tayuetateke@gmail.com(T. Tateke)

\section{To cite this article:}

Tayue Tateke, MirkuzieWoldie, ShimelesOlolo. Determinants of Perceived Health Care Provider Empathy at Public and Private Hospitals in Central Ethiopia. Science Journal of Public Health. Vol. 1, No. 3, 2013, pp. 156-164. doi: 10.11648/j.sjph.20130103.18

\begin{abstract}
Introduction:Though being truly empathic with the patient in health service provision is challenging, it is a powerful and efficient communication tool when used appropriately. It is valuable for healthcare providers and receivers if patients perceived the healthcare providers understand the values, ideas and feelings of their patients. Therefore, this study was carried out to measure the level of perceived healthcare provider empathy and its' determinants among outpatients of public and private hospitals in Addis Ababa, central Ethiopia.Material and Methods: Data were collected as part of a comparative cross sectional study conducted during March 27 to April 30/2010 in Addis Ababa. The size of the sample was determined using double population proportion formula. The study participants wereoutpatients who were identified using systematic sampling technique at randomly selected private and public hospitals. The data were collected using a structured questionnaire with Likert scales to measure perception of patients. Data were entered into computer software (SPSS version 16.0) to generate descriptive statistics and factor scores. ANOVA and multiple linear regression analysis were conducted.Result: The respondents of the study were 626 (313 for each group of the hospitals) with response rate of 93.71\%.The mean ( \pm S.D) levels of perceived empathy at public and private hospitals were $33.47( \pm 11.654)$ and $38.16( \pm$ 10.161), respectively with possible value range of 10 to 50. At public hospitals, Welcoming approach, body signaling, consultation duration, perceived providers' technical competency, perceived providers lack of experience and ability and expectation about the hospital services were appeared significant determinants of perceived empathy level. Likewise, perceived welcoming approach, body signaling, perceived providers technical competency, perceived providers lack of experience and ability, waiting time, perceived accessibility, expectation about hospital services and perceived cleanliness predicted perceived empathy at private hospitals.Conclusion: Despite significant difference in the level of perceived empathy at public and private hospitals, four of the identified determinants were common. The predictors of perceived provider empathy in this study were related to characteristics of patients, health care providers and the health system. Therefore, health service managers and health care providers should work on improving the communication skills of health professionals which could significantly alter the perception of the patient to services s/he receives at the outpatient department.
\end{abstract}

Keywords: Healthcare, Empathy, Treatment

\section{Introduction}

In health service provision, empathy holds an important position for efficient and effective communication (1-4). It helps the service providers to understand the value, ideas and feelings of patients beyond history and symptoms understandings. On the other hand, the perception of patients on their health care providers' empathy has effect on how they make their health care decisions and on their satisfaction(5).Patients who see positive characteristics in their providers (such as being empathic) and feel comfort of being understood are more likely to seek treatment and follow medical advices (6). Thus, though being truly empathic is challenging as it is a complex and multifaceted construct,itsbenefit for both physician and patients has been evidenced with the previous studies(7-16).

Since the last two decades, the healthcare delivery system in Ethiopia is witnessing a rapid proliferation of private healthcare providers including hospitals of advanced care though mainly limited to urban centers such 
as the capital Addis Ababa. This has been a response to the policy direction of the current government to increase access and quality of healthcare received by the people. As a result,thetwo categories of hospitals, public and private, are operating with differences in regulatory framework and other characteristics which could possibly cause differences in the process of service provision. More specifically, the manner in which services are being provided in these groups of hospitals isdifferent sincethe technologies, the physical structure, the management process and others they operate in are quite dissimilar despite the expected improvement from the recent government hospitals' business process reengineering (BPR) implementation.

Health care providers in both categories of hospitals usually meet with patients with different needs and personal characters. The interaction here is not affected only by what the health care provider do, the perception of the patients as to how the health care providers treat them with empathy is highly important.Hence, both verbal and non-verbal communication cues used by the healthcare providers during clinical encountersare likely to be interpreted differently. Moreover, this perception can be affected by different factors (17-24). Thus, identifying category specific factors that determine the level of perceived health care providers' empathy at the public and private hospitals will be helpful in improving the quality of health care provider-patient interaction. Therefore, this study was conducted to measure and compare the level of perceived healthcare provider empathy among outpatients of public and private hospitals and identify category specific determinants.

\section{Methods}

\subsection{Study Design and Participants}

Data were collected as part of a comparative cross sectional study conducted during March 27 to April 30/2010 in Addis Ababa, central Ethiopia (25).

The total sample of patients to be interviewed was determined to be 668 using double population proportion formula. The study participants were identified using systematic random sampling at the OPDs of 10 hospitals in Addis Ababa(5 private and 5 public)from those outpatients who visited during the study period. Number of respondents from each hospital was determined based on the proportion of patients who visited the OPDs during one month prior to the start of the study. The interval for including a patient in the study was determined by dividing the total number of patients at the private or public hospitals in the last one month by the sample size for each category of hospitals. During the interview, critically ill patients were excluded unless they had a care taker who was willing to respond and parents or care givers of children were interviewed at the pediatric OPDs.

\subsection{Data Collection Instrument and Procedures}

The data were collected using a structured questionnaire prepared based on tools and constructs used in earlier similar studies $(26,27)$. The questionnaire was developed in English and translated into Amharic (the local language) and back retranslated into English to ensure consistency. Then the instrument was pre-tested to check appropriateness of wording, format, length, sequencing, and the range of the scales for Likert scale questions. Multiple items were used to establish appropriate measurement properties (reliability and validity) of the selected constructs. The participants were interviewed by trained data collectors after using the services andimmediately before their exitfrom the hospital compound. Consultation duration was recorded by observingthe time patients spent at the examination room, from entry to exit.

\subsection{Data Analysis}

Using SPSS version 16.0 descriptive statistics was used for determining indices. Factor analysis was carried out to identify factors that explain most of the variance observed in the population with regard to each scale. Analysis of variance for comparing responses from public and private hospital respondents and multiple linear regressions for determining factors predicting perceived healthcare provider empathy were performed. A significance level of 0.05 was used in all cases.

\subsection{Ethical Considerations}

All the necessary procedures to ensure autonomy of the patient were followed during data collection as outlined in an earlier article (25).

\section{Results}

\subsection{Characteristics of the Respondents}

A total of 626 outpatients (313 from each group of the hospitals) were interviewed yielding a response rate of 93.71\%. At the public hospitals, the median age of the respondents was 30 years with range of 15-75 while it was 32 years with range of 14- 75 for the respondents from the private hospitals. Further details on the socio-demographic characteristics of the respondents were reported in an earlier article (25).

\subsection{Measuring perceived empathy}

The mean $( \pm$ S.D) levels of perceived healthcare provider empathy at public and private hospitals were 33.47 ( \pm $11.654)$ and $38.16( \pm 10.161)$, respectively with possible value range of 10 to 50 . The difference of the means was significant with $(\mathrm{F}[624 ; 625]=59.201, p \leq 0.000)$. Moreover, three factors were extracted from a scale of ten items (Cronbach's alpha value of 0.855) to measure perceived empathy among outpatients of private hospitals. 
These factors explained $52.69 \%$ of the variance among those patients. While at public hospitals, $61.37 \%$ of the variance among the patients' perception was explained by three factors extracted from a scale of 8 items with Cronbach's alpha value of 0.871 . Two of the items were excluded in this case sincethey loaded on two factors simultaneously.

The items loaded on factor one and three were similar for both private and public hospitals but on the second factor the items were different. We tried to give name for each of the factors identified to ease the understanding of the findings in this paper. Accordingly, for both categories of hospitals, items loaded on the first factor were: letting the patient tell his/herstory,really listening while they are talking and making them feel at ease. This factor was named as "perceived listening ability". On the second factor, showing care and compassion, being positive and being interested as a whole person were included for public hospitals. We named this factor as "perceived caring behavior". For the private hospitals, the loaded items were: showing care and compassion, being positive, explaining things clearly and fully understand the patients concernwhich was named as "perceived caring and understanding behavior". Forthe third factor, helping the patient to take control and involving them in decisions were the items included for both categories of hospitals. We named this group as "perceived empowering behavior".

\subsection{Predictors of Empathy}

\subsubsection{Social back Ground and Previous Service Experience as Predictor}

Among outpatients ofthe private hospitals, ethnicity and religion showed statistically significant association with perceived listing ability. For instance, respondents from Oromo ethnicity had 0.475 (95\% CI, $0.188-0.762)$ unit higher perceived listening ability score as compared to respondents fromthe Amhara ethnic group. Similarly, for perceived caring and understanding behavior statistically significant association was found withmarital status and occupation. Accordingly, single respondents had $0.324(95 \%$, CI: 0.600 to -0.048$)$ units lower perceived caring and understanding behavior score as compared to themarried respondents. Wealth index was found to be the only socio demographic variable having statistically significant associationwith perceived empowering behavior (Table1).

Table 1. Socio demographic predictors of perceived empathy at private hospitals in Addis Ababa, central Ethiopia, 2010

\begin{tabular}{|c|c|c|c|c|c|c|c|c|c|}
\hline \multirow{2}{*}{ Factors } & \multirow{2}{*}{ Variables } & & \multirow{2}{*}{$\begin{array}{l}\text { Frequency } \\
(\%)\end{array}$} & \multicolumn{2}{|c|}{$\begin{array}{l}\text { Un standardized } \\
\text { Coefficients }\end{array}$} & \multirow[t]{2}{*}{$\begin{array}{l}\text { Standardized } \\
\text { Coefficients }\end{array}$} & \multirow{2}{*}{$\begin{array}{l}\text { p- } \\
\text { value }\end{array}$} & \multicolumn{2}{|c|}{$\begin{array}{l}\text { 95\% Confidence Interval for } \\
\text { B }\end{array}$} \\
\hline & & & & & $\begin{array}{l}\text { Std. } \\
\text { Error }\end{array}$ & & & $\begin{array}{l}\text { Lower } \\
\text { Bound }\end{array}$ & $\begin{array}{l}\text { Upper } \\
\text { Bound }\end{array}$ \\
\hline \multirow{9}{*}{$\begin{array}{l}\text { Perceived } \\
\text { listening } \\
\text { ability }\end{array}$} & \multirow{4}{*}{ Religion } & Orthodox* & $193(61.7)$ & & & & & & \\
\hline & & Muslim & 61(19.3) & -.307 & .156 & -.134 & .050 & -.614 & .000 \\
\hline & & Protestant & $49(15.7)$ & -.262 & .161 & -.105 & .105 & -.580 & .055 \\
\hline & & Others** & $10(3.2)$ & .033 & .324 & .006 & .919 & -.604 & .670 \\
\hline & \multirow{6}{*}{ Ethnicity } & Amhara* & $133(42.5)$ & & & & & & \\
\hline & & Oromo & $64(20.4)$ & .475 & .146 & .212 & .001 & .188 & .762 \\
\hline & & Tigre & $28(8.9)$ & .038 & .153 & .016 & .803 & -.263 & .340 \\
\hline & & Gurage & $55(17.6)$ & .077 & .193 & .024 & .692 & -.303 & .456 \\
\hline & & Others*** & $33(10.6)$ & -.192 & .564 & -.021 & .734 & -1.303 & .918 \\
\hline \multirow{12}{*}{$\begin{array}{l}\text { Perceived } \\
\text { caring and } \\
\text { understandin } \\
\mathrm{g} \text { behavior }\end{array}$} & & Married* & $185(59.2)$ & & & & & & \\
\hline & \multirow{3}{*}{$\begin{array}{l}\text { Marital } \\
\text { status }\end{array}$} & Single & $115(36.7)$ & -.324 & .140 & -.176 & .022 & -.600 & -.048 \\
\hline & & Divorced & $2(0.6)$ & .256 & .649 & .023 & .693 & -1.021 & 1.532 \\
\hline & & Widowed & $11(3.5)$ & .343 & .299 & .071 & .253 & -.246 & .932 \\
\hline & \multirow{8}{*}{$\begin{array}{l}\text { Occupati } \\
\text { on }\end{array}$} & Gov’t employee* & $71(22.7)$ & & & & & & \\
\hline & & Merchant & $69(22.0)$ & -.378 & .170 & -.177 & .027 & -.712 & -.043 \\
\hline & & NGO employee & $68(21.7)$ & -.213 & .157 & -.099 & .177 & -.523 & .096 \\
\hline & & Housewife & $50(16.0)$ & -.399 & .207 & -.165 & .055 & -.806 & .009 \\
\hline & & Daily laborer & $5(1.6)$ & -.181 & .462 & -.026 & .696 & -1.090 & .729 \\
\hline & & Student & $28(8.9)$ & -.186 & .228 & -.060 & .416 & -.636 & .263 \\
\hline & & Farmer & $7(2.2)$ & .188 & .395 & .031 & .635 & -.590 & .965 \\
\hline & & Others $* * * *$ & $15(4.8)$ & -.282 & .281 & -.068 & .316 & -.835 & .271 \\
\hline $\begin{array}{l}\text { Perceived } \\
\text { empowering }\end{array}$ & $\begin{array}{l}\text { Economi } \\
\text { c status }\end{array}$ & Wealth index & & .034 & 017 & .131 & .046 & .001 & .066 \\
\hline
\end{tabular}

*Reference group $* *$ includes catholic and traditional religion follower

*** includes Somalie, Wolayta, Hadya, Afar **** includes taxi driver, guard man, broker

At public hospitals, protestant respondents reported 0.383 and 0.347 (95\% CI:-0.728 to -0.037 and -0.658 to -0.036 ) units lower perceived listing ability and perceived empowering scores, respectively,as compared to
theirOrthodox Christiancounterparts. But perceived caring behavior score was not associated with any of the socio demographic variables (Table 2). 
Table 2.Socio demographic predictors of perceived empathy at public hospitals in Addis Ababa, central Ethiopia, 2010

\begin{tabular}{|c|c|c|c|c|c|c|c|c|c|}
\hline \multirow{2}{*}{ Factors } & \multirow{2}{*}{ Variables } & & \multirow{2}{*}{$\begin{array}{l}\text { Frequency } \\
(\%)\end{array}$} & \multicolumn{2}{|c|}{$\begin{array}{l}\text { Unstandardized } \\
\text { Coefficients }\end{array}$} & \multirow{2}{*}{$\begin{array}{l}\text { Standardized } \\
\text { Coefficients } \\
\text { Beta }\end{array}$} & \multirow{2}{*}{$\begin{array}{l}P \text { - } \\
\text { value }\end{array}$} & \multicolumn{2}{|c|}{$\mathbf{9 5 \%}$ Confidence Interval for B } \\
\hline & & & & B & Std. Error & & & Lower Bound & Upper Bound \\
\hline \multirow{4}{*}{$\begin{array}{l}\text { Perceived } \\
\text { listening } \\
\text { ability }\end{array}$} & \multirow{4}{*}{ Religion } & Orthodox* & 193(61.7) & & & & & & \\
\hline & & Muslim & $61(19.3)$ & -.057 & .162 & -.022 & .728 & -.376 & .263 \\
\hline & & Protestant & $49(15.7)$ & -.383 & .176 & -.134 & .030 & -.728 & -.037 \\
\hline & & Others** & $10(3.2)$ & .058 & .648 & .005 & .929 & -1.218 & 1.334 \\
\hline \multirow{4}{*}{$\begin{array}{l}\text { Perceived } \\
\text { empowering } \\
\text { behavior }\end{array}$} & \multirow{4}{*}{ Religion } & Orthodox* & 193(61.7) & & & & & & \\
\hline & & Muslim & $61(19.3)$ & .016 & .146 & .007 & .912 & -.271 & .304 \\
\hline & & Protestant & $49(15.7)$ & -.347 & .158 & -.135 & .029 & -.658 & -.036 \\
\hline & & Others** & $10(3.2)$ & .081 & .582 & .008 & .889 & -1.065 & 1.228 \\
\hline
\end{tabular}

*Reference group** includes catholic, traditional religion follower

Expectation aboutthe hospital services was significantly associated with all of the three factorsof perception atprivate hospitals. Respondents who had low expectation about the health services reported0.514units lower perceived listening abilityas compared to those with high expectation (95\% CI: -1.011 to -0.016$)$.Similarly, a reduction of 0.415 units was observed on the perceived empowering behavior score of patients with low expectation about the health services provided, (95\% CI:0.828 to -0.001 ). On the other hand, a standard deviation additional minute on recorded consultation duration raised the perceived caring and understanding behavior score by $0.193(p \leq 0.000)$ standard deviations. The corresponding increment for perceived empowering behavior score was $0.180(p=0.000)$ standard deviations (Table 3$)$.

Table 3.Previous patient experiences as predictors of perceived empathy at private hospitals in Addis Ababa, central Ethiopia, 2010

\begin{tabular}{|c|c|c|c|c|c|c|c|c|}
\hline \multirow{2}{*}{ Factors } & \multirow{2}{*}{ Variables } & \multirow{2}{*}{$\begin{array}{l}\text { Frequenc } \\
\text { y (\%) }\end{array}$} & \multicolumn{2}{|c|}{$\begin{array}{l}\text { Unstandardized } \\
\text { Coefficients }\end{array}$} & \multirow{2}{*}{$\begin{array}{l}\text { Standardized } \\
\text { Coefficients } \\
\text { Beta }\end{array}$} & \multirow{2}{*}{$\begin{array}{l}\text { P- } \\
\text { value }\end{array}$} & \multicolumn{2}{|c|}{$\mathbf{9 5 \%}$ Confidence Interval for B } \\
\hline & & & B & $\begin{array}{l}\text { Std. } \\
\text { Error }\end{array}$ & & & Lower Bound & Upper Bound \\
\hline \multirow{5}{*}{$\begin{array}{l}\text { Perceived } \\
\text { listening } \\
\text { ability }\end{array}$} & Expectation abouttheservice & & & & & & & \\
\hline & High* & $154(49.2)$ & & & & & & \\
\hline & Medium & $120(38.3)$ & -.034 & .114 & -.018 & .764 & -.259 & .191 \\
\hline & Low & $14(4.5)$ & -.514 & .253 & -.117 & .043 & -1.011 & -.016 \\
\hline & None & $25(8.0)$ & -.513 & .201 & -.154 & .011 & -.909 & -.118 \\
\hline \multirow{7}{*}{$\begin{array}{l}\text { Perceived } \\
\text { caring and } \\
\text { understandin } \\
\text { g behavior }\end{array}$} & Consultation duration & & .029 & .008 & .193 & .000 & .013 & .044 \\
\hline & History of admission & & -.317 & .147 & -.122 & .033 & -.607 & -.026 \\
\hline & Expectation about the service & & & & & & & \\
\hline & High* & $154(49.2)$ & & & & & & \\
\hline & Medium & $120(38.3)$ & -.481 & .106 & -.263 & .000 & -.689 & -.273 \\
\hline & Low & $14(4.5)$ & -.469 & .234 & -.109 & .046 & -.930 & -.009 \\
\hline & None & $25(8.0)$ & -.823 & .186 & -.251 & .000 & -1.189 & -.456 \\
\hline \multirow{6}{*}{$\begin{array}{l}\text { Perceived } \\
\text { empowering } \\
\text { behavior }\end{array}$} & Consultation duration & & .023 & .007 & .180 & .002 & .009 & .037 \\
\hline & Expectation about the service & & & & & & & \\
\hline & High* & $154(49.2)$ & & & & & & \\
\hline & Medium & $120(38.3)$ & .008 & .095 & .005 & .937 & -.179 & .194 \\
\hline & Low & $14(4.5)$ & -.415 & .210 & -.114 & .049 & -.828 & -.001 \\
\hline & None & $25(8.0)$ & .132 & .167 & .048 & .429 & -.197 & .461 \\
\hline
\end{tabular}

*Reference group

At public hospitals, respondents who judged their health status as "no change" reported 0.332 units lower perceived caring behaviorscore as compared to those who judged their health status as "well" ( $95 \% \mathrm{CI}:-0.577$ to -0.087$)$. Similarly, 0.367 units lower empowering behaviors scores were reported by patients who judged their status as "no change" (95\%CI: -0.589 to -0.145$)$. Besides, perceived caring behavior score of patients with low expectation aboutservices at public hospitals wasdecreased by 0.477 units as compared to those with high expectation (95\% CI, -0.790 to -0.163 ). Perceived listening ability was associated with none of the variables (Table 4). 
Table 4.Previous patient experiences as predictors of perceived empathy at public hospitals in Addis Ababa, central Ethiopia, 2010

\begin{tabular}{|c|c|c|c|c|c|c|c|c|}
\hline \multirow{2}{*}{ Factors } & \multirow{2}{*}{ Variables } & \multirow{2}{*}{$\begin{array}{l}\text { Frequency } \\
(\%)\end{array}$} & \multicolumn{2}{|c|}{$\begin{array}{l}\text { Unstandardized } \\
\text { Coefficients }\end{array}$} & \multirow{2}{*}{$\begin{array}{l}\text { Standardized } \\
\text { Coefficients } \\
\text { Beta }\end{array}$} & \multirow{2}{*}{$\begin{array}{l}\text { P- } \\
\text { value }\end{array}$} & \multicolumn{2}{|c|}{ 95\% Confidence Interval for B } \\
\hline & & & B & Std. Error & & & Lower Bound & Upper Bound \\
\hline \multirow{10}{*}{$\begin{array}{l}\text { Perceived } \\
\text { caring } \\
\text { behavior }\end{array}$} & Expectation aboutthe service & & & & & & & \\
\hline & High & $114(36.42)$ & -.208 & .117 & -.115 & .078 & -.439 & .023 \\
\hline & Medium* & $126(40.3)$ & & & & & & \\
\hline & Low & $45(14.4)$ & -.477 & .159 & -.188 & .003 & -.790 & -.163 \\
\hline & None & $28(8.9)$ & -.210 & .190 & -.067 & .270 & -.584 & .164 \\
\hline & Self-judged health status & & & & & & & \\
\hline & Verywell & $38(12.1)$ & .256 & .162 & .094 & .116 & -.064 & .575 \\
\hline & Well* & $158(50.48)$ & & & & & & \\
\hline & Nochange & $81(25.9)$ & -.332 & .125 & -.164 & .008 & -.577 & -.087 \\
\hline & Gettingworse & $36(11.5)$ & -.184 & .167 & -.066 & .271 & -.514 & .145 \\
\hline \multirow{5}{*}{$\begin{array}{l}\text { Perceived } \\
\text { empowerin } \\
\text { g behavior }\end{array}$} & Self-judged health status & & & & & & & \\
\hline & Verywell & $38(12.1)$ & .370 & .147 & .149 & .012 & .081 & .659 \\
\hline & Well* & $158(50.48)$ & & & & & & \\
\hline & Nochange & $81(25.9)$ & -.367 & .113 & -.198 & .001 & -.589 & -.145 \\
\hline & Gettingworsen & $36(11.5)$ & -.293 & .152 & -.115 & .054 & -.592 & .005 \\
\hline
\end{tabular}

*Reference group

\subsubsection{Perceived Service Characteristics as Predictors}

At publichospitals, a standard deviation riseinthe score of perceivedwelcoming approach increased the perceived listening abilityand caring behavior scoresby $0.404(\mathrm{p} \leq$ $0.000)$ and $0.300 \quad(p \leq 0.000)$ standard deviations, respectively. Likewise, the perceived empowering behavior score showed riseof $0.344(\mathrm{p} \leq 0.000)$ standard deviations when theperceived provider's technical competency score increased by one standard deviation. However, a standard deviation increment in perceived lack of experience and ability score lowered the perceived empowering behavior score by 0.122 standarddeviations $(\mathrm{p}=0.019)$. Surprisingly,a standard deviation increment in perceived cost of the public hospital services raised the perceived listening ability by $0.117(\mathrm{p}=0.027)$ standard deviations(Table 5).

Table 5.Perceived service characteristics as predictors of perceived empathy at public hospitals in Addis Ababa, central Ethiopia, 2010

\begin{tabular}{|c|c|c|c|c|c|c|c|}
\hline \multirow{2}{*}{ Factors } & \multirow{2}{*}{ Variables } & \multicolumn{2}{|c|}{$\begin{array}{l}\text { Unstandardized } \\
\text { Coefficients }\end{array}$} & \multirow{2}{*}{$\begin{array}{l}\text { Standardized } \\
\text { Coefficients } \\
\text { Beta }\end{array}$} & \multirow{2}{*}{$\begin{array}{l}\text { P- } \\
\text { value }\end{array}$} & \multicolumn{2}{|c|}{ 95\% Confidence Interval for B } \\
\hline & & B & Std. Error & & & Lower Bound & Upper Bound \\
\hline \multirow{4}{*}{$\begin{array}{l}\text { Perceived } \\
\text { listening } \\
\text { ability }\end{array}$} & Perceived cleanliness of public hospitals & .146 & .049 & .154 & .003 & .049 & .243 \\
\hline & Perceived welcoming approach & .399 & .058 & .404 & .000 & .285 & .513 \\
\hline & Perceived body signaling & .123 & .056 & .112 & .028 & .013 & .233 \\
\hline & Perceived cost for public hospitals & .113 & .051 & .117 & .027 & .013 & .212 \\
\hline \multirow{4}{*}{$\begin{array}{l}\text { Perceived } \\
\text { caring } \\
\text { behavior }\end{array}$} & Perceived providers technical competency & .129 & .055 & .137 & .020 & .020 & .238 \\
\hline & Perceived welcoming approach & .293 & .059 & .300 & .000 & .178 & .408 \\
\hline & Perceived accessibility for public hospitals & .193 & .059 & .179 & .001 & .077 & .309 \\
\hline & Perceived length of waiting time & .160 & .062 & .137 & .010 & .039 & .281 \\
\hline \multirow{3}{*}{$\begin{array}{l}\text { Perceived } \\
\text { empoweri } \\
\text { ng } \\
\text { behavior }\end{array}$} & Perceived providers technical competency & .296 & .050 & .344 & .000 & .197 & .395 \\
\hline & $\begin{array}{l}\text { Perceived providers lack of experience and } \\
\text { ability }\end{array}$ & -.108 & .046 & -.122 & .019 & -.199 & -.018 \\
\hline & Perceived body signaling & .150 & .052 & .152 & .004 & .049 & .252 \\
\hline
\end{tabular}

At private hospitals, a standard deviation increment in perceived providers' welcoming approach score raised perceived listening ability score by $0.280(p \leq 0.000)$ and perceived caring and understanding behavior by $0.339(\mathrm{p} \leq$ 0.000 ) standard deviations. A standard deviation increment in perceived providers' technical competency raised perceived caring and understanding score by $0.287(\mathrm{p} \leq$ 0.000 ) standard deviations. Nevertheless, one standard deviation increment in perceived providers'lack of experience and ability causedthe perceived caring and understanding behavior score to be lowered by $0.132(\mathrm{p}=$ 0.010) standard deviations(Table 6). 
Table 6.Perceived service characteristics as predictors of perceived empathy at private hospitals in Addis Ababa, central Ethiopia, 2010

\begin{tabular}{|c|c|c|c|c|c|c|c|}
\hline \multirow{2}{*}{ Factors } & \multirow{2}{*}{ Variables } & \multicolumn{2}{|c|}{$\begin{array}{l}\text { Unstandardized } \\
\text { Coefficients }\end{array}$} & \multirow{2}{*}{$\begin{array}{l}\text { Standardized } \\
\text { Coefficients } \\
\text { Beta }\end{array}$} & \multirow{2}{*}{$\begin{array}{l}P \text { - } \\
\text { value }\end{array}$} & \multicolumn{2}{|c|}{$\mathbf{9 5 \%}$ Confidence Interval for $\mathrm{B}$} \\
\hline & & B & $\begin{array}{l}\text { Std. } \\
\text { Error }\end{array}$ & & & Lower Bound & Upper Bound \\
\hline Perceived & Perceived welcoming approach & .272 & .058 & .280 & .000 & .157 & .387 \\
\hline listening & Perceived open posture & .240 & .067 & .198 & .000 & .109 & .371 \\
\hline ability & Perceived private hospitals cost & .107 & .053 & .108 & .046 & .002 & .211 \\
\hline \multirow{4}{*}{$\begin{array}{l}\text { Perceived } \\
\text { caring and } \\
\text { understanding } \\
\text { behavior }\end{array}$} & Perceived welcoming approach & .323 & .051 & .339 & .000 & .222 & .423 \\
\hline & Perceived open posture & .145 & .058 & .122 & .013 & .030 & .259 \\
\hline & Perceived providers technical competency & .278 & .050 & .287 & .000 & .180 & .375 \\
\hline & $\begin{array}{l}\text { Perceived providers lack of experience and } \\
\text { ability }\end{array}$ & -.131 & .050 & -.132 & .010 & -.230 & -.032 \\
\hline $\begin{array}{l}\text { Perceived } \\
\text { empowering } \\
\text { behavior }\end{array}$ & Perceived providers technical competency & .172 & .050 & .208 & .001 & .074 & .269 \\
\hline
\end{tabular}

\subsubsection{Independent Predictors of Perceived Empathy}

Several variables appeared significant to determine perceived provider empathy at both private and public hospitals. At private hospitals, a standard deviation increment in the score of perceived welcoming approach raised perceived listening ability score by $0.266(\mathrm{P} \leq 0.000)$ and perceived caring and understanding behavior score by $0.290(\mathrm{P} \leq 0.000)$ standard deviations. Likewise, a standard deviation increment in score of perceived body signaling raised perceived listening ability score by $0.185(\mathrm{p}=0.001)$ and perceived caring and understanding behavior score by $0.129(p=0.008)$ standard deviations. Respondents who had "no expectation"about the hospital services had $0.433(95 \%$ CI:-0.758 to -0.107) units lower perceived provider caring and understanding behavior compared to those who had "high" expectation for the hospital services (Table 7).

Table 7.Final predictors of perceived empathy at private hospitals in Addis Ababa, central Ethiopia, 2010

\begin{tabular}{|c|c|c|c|c|c|c|c|c|}
\hline \multirow[t]{2}{*}{ Factors } & \multirow[t]{2}{*}{ Variables } & \multirow{2}{*}{$\begin{array}{l}\text { Frequenc } \\
\text { y }(\%)\end{array}$} & \multicolumn{2}{|c|}{$\begin{array}{l}\text { Unstandardized } \\
\text { Coefficients }\end{array}$} & \multirow{2}{*}{$\begin{array}{l}\text { Standardized } \\
\text { Coefficients } \\
\text { Beta }\end{array}$} & \multirow{2}{*}{$\begin{array}{l}\text { P- } \\
\text { value }\end{array}$} & \multicolumn{2}{|c|}{$\mathbf{9 5 \%}$ Confidence Interval for B } \\
\hline & & & B & Std. Error & & & Lower Bound & Upper Bound \\
\hline \multirow{9}{*}{$\begin{array}{l}\text { Perceived } \\
\text { listening } \\
\text { ability }\end{array}$} & Ethnicity & & & & & & & \\
\hline & Amhara* & $133(42.5)$ & & & & & & \\
\hline & Oromo & $64(20.4)$ & .463 & .127 & .206 & .000 & .213 & .713 \\
\hline & Tigre & $28(8.9)$ & .024 & .134 & .010 & .857 & -.239 & .288 \\
\hline & Gurage & $55(17.6)$ & -.029 & .174 & -.009 & .868 & -.372 & .314 \\
\hline & Others*** & $33(10.6)$ & .110 & .510 & .012 & .830 & -.893 & 1.113 \\
\hline & Perceived welcoming approach & & .259 & .054 & .266 & .000 & .153 & .364 \\
\hline & Perceived open posture & & .225 & .066 & .185 & .001 & .095 & .354 \\
\hline & Consultation duration & & .021 & .007 & .144 & .003 & .007 & .035 \\
\hline \multirow{8}{*}{$\begin{array}{l}\text { Perceived } \\
\text { caring and } \\
\text { understand } \\
\text { ing } \\
\text { behavior }\end{array}$} & $\begin{array}{l}\text { Expectation about the services } \\
\text { High* }\end{array}$ & $154(49.2)$ & & & & & & \\
\hline & Medium & $120(38.3)$ & -.175 & .100 & -.096 & .080 & -.371 & .021 \\
\hline & Low & $14(4.5)$ & -.162 & .211 & -.038 & .444 & -.578 & .254 \\
\hline & None & $25(8.0)$ & -.433 & .165 & -.132 & .009 & -.758 & -.107 \\
\hline & Perceived welcoming approach & & .276 & .052 & .290 & .000 & .175 & .378 \\
\hline & Perceived open posture & & .154 & .057 & .129 & .008 & .041 & .267 \\
\hline & $\begin{array}{l}\text { Perceived providers technical } \\
\text { competency }\end{array}$ & & .226 & .051 & .233 & .000 & .124 & .327 \\
\hline & $\begin{array}{l}\text { Perceived providers lack or } \\
\text { experience and ability }\end{array}$ & & -.103 & .050 & -.104 & .041 & -.202 & -.004 \\
\hline \multirow{2}{*}{$\begin{array}{l}\text { Perceived } \\
\text { empoweri } \\
\text { ng } \\
\text { behavior }\end{array}$} & Consultation duration & & .020 & .007 & .160 & .004 & .006 & .034 \\
\hline & $\begin{array}{l}\text { Perceived providers technical } \\
\text { competency }\end{array}$ & & .193 & .048 & .235 & .000 & .098 & .288 \\
\hline
\end{tabular}

*Reference group*** includes Somalie, Wolaita, Hadya, Afar

At public hospitals, a standard deviation additional score in perceived welcoming approach resulted in 0.439 ( $p \leq$ $0.000)$ and $0.277(\mathrm{p} \leq 0.000)$ standard deviations increment in perceived listening ability and caring behavior scores, respectively.On the other hand, a standard deviation additional score in perceived cleanliness raised the perceived listening ability score by $0.168(\mathrm{p}=0.001)$ standard deviations. Along with this, respondents who had low expectation for hospital services had 0.298(95\% CI, 0.582 to -0.015$)$ units lowered perceived caring behavior score than those respondents with high expectation (Table $8)$. 
Table 8. Final predictors of perceived empathy at public hospitals in Addis Ababa, central Ethiopia, 2010

\begin{tabular}{|c|c|c|c|c|c|c|c|c|}
\hline \multirow{2}{*}{ Factors } & \multirow{2}{*}{ Variables } & \multirow{2}{*}{$\begin{array}{l}\text { Frequency } \\
\text { (\%) }\end{array}$} & \multicolumn{2}{|c|}{$\begin{array}{l}\text { Unstandardized } \\
\text { Coefficients }\end{array}$} & \multirow{2}{*}{$\begin{array}{l}\text { Standardized } \\
\text { Coefficients } \\
\text { Beta }\end{array}$} & \multirow{2}{*}{$\begin{array}{l}\text { P- } \\
\text { value }\end{array}$} & \multicolumn{2}{|c|}{$\begin{array}{l}95 \% \text { Confidence } \\
\text { Interval for B }\end{array}$} \\
\hline & & & B & $\begin{array}{l}\text { Std. } \\
\text { Error }\end{array}$ & & & $\begin{array}{l}\text { Lower } \\
\text { Bound }\end{array}$ & $\begin{array}{l}\text { Upper } \\
\text { Bound }\end{array}$ \\
\hline \multirow{4}{*}{$\begin{array}{l}\text { Perceived } \\
\text { listening } \\
\text { ability }\end{array}$} & Perceived cleanliness of hospitals & & .160 & .048 & .168 & .001 & .066 & .253 \\
\hline & Perceived welcoming approach & & .433 & .052 & .439 & .000 & .331 & .536 \\
\hline & Perceived body signaling & & .113 & .055 & .104 & .040 & .005 & .222 \\
\hline & $\begin{array}{l}\text { Expectation for the hospital services } \\
\text { High } \\
\text { Medium* }\end{array}$ & $\begin{array}{l}114(36.42) \\
126(40.3)\end{array}$ & -.161 & .104 & -.089 & .123 & -.366 & .044 \\
\hline \multirow{6}{*}{$\begin{array}{l}\text { Perceived } \\
\text { caring } \\
\text { behavior }\end{array}$} & Low & $45(14.4)$ & -.298 & .144 & -.118 & .039 & -.582 & -.015 \\
\hline & None & $28(8.9)$ & -.049 & .171 & -.016 & .775 & -.385 & .287 \\
\hline & $\begin{array}{l}\text { Perceived providers technical } \\
\text { competency }\end{array}$ & & .124 & .055 & .132 & .024 & .017 & .232 \\
\hline & Perceived welcoming approach & & .270 & .060 & .277 & .000 & .152 & .389 \\
\hline & Perceived accessibility for hospitals & & .185 & .058 & .171 & .002 & .071 & .298 \\
\hline & Perceived length of waiting time & & .187 & .061 & .160 & .002 & .068 & .307 \\
\hline \multirow{3}{*}{$\begin{array}{l}\text { Perceived } \\
\text { empowering } \\
\text { behavior }\end{array}$} & $\begin{array}{l}\text { Perceived providers technical } \\
\text { competency }\end{array}$ & & .293 & .045 & .341 & .000 & .205 & .382 \\
\hline & $\begin{array}{l}\text { Perceived providers lack of } \\
\text { experience and ability }\end{array}$ & & -.113 & .044 & -.127 & .012 & -.200 & -.025 \\
\hline & Perceived body signaling & & .146 & .051 & .147 & .005 & .045 & .246 \\
\hline
\end{tabular}

*Reference group

\section{Discussion}

The level of perceived health care providers' empathy is the result of the dynamics of negative and positive effect communication and treatment factors. Therefore, to lessen the negative effects and to add on the positive effects of these factors, different strategies might be used. However, this requires the identification of all context specific factors. In the current study, despite the recent implementation of the all rounded business process reengineering (BPR) in public hospitals, the level of perceived health care providers' empathy was relatively lower than private hospitals. However, the levels at both categories of hospitalswere higher than the finding in primary health care centers of central Ethiopia andlower than other findings reported fromelsewhere $(14,17,19-22)$. The difference of the current score from the previous finding in Ethiopia can partly be explained by patients' expectation for services at primary health care centers and hospitals and the socio demographic differences of urban and rural residents. On the other hand, the difference of the finding from the other findings can be because of the differences in study settings and socio demographics of respondents.

In the present study, different forms of non-verbal communications cues affectedpatients' perception for their health care providers' empathy. Especially at private hospitals, theeffect of non- verbal communication was significant for two of the factors extracted from the scale used to measure perceived provider empathy: perceived listening ability and perceived caring and understanding behavior. The same was true for the factors which emerged at the public hospitals survey with perceived listening ability taking the lead. This indicates how patients are attentive tothe non-verbal communication cuesdemonstrated by the health care provider. Non-verbal communication has also been identified as an important predictor of perceived providers' empathy toward the patient in other studies $(17,24)$.However, it has to be noted that these studies described non-verbal communication grossly while its different aspects were considered separately in the current study to highlight on the relative importance of the different dimensions on perceived empathy. This helps to demonstrate the degree of emphasis health managers and health care providers should give to the different aspects of non-verbal communication in their efforts to improve patient-provider interaction in different settings.

The other factor that affected most of the perceived empathy factors in this study was health care perceived providers' technical competency. The finding is consistent with other finding reported earlier (17). It was found that perceived providers' technical competency predicts two (perceived caring and empowering behavior) of the factors extracted from the empathy scale among patients of the public hospitals. While perceived providers' technical competency did not affect perceived caring behavior among the respondents from private hospitals, it predicted the extent of perceived caring and understanding behavior and empowering behavior. More specifically, it was noted that perceived providers' technical competency has positive effect on perceived level of empathy while perceived providers' lack of experience and ability hadnegative effect.

On the other hand, among the patients' characteristics, ethnicity appeared to be determinant for perceived health care providers' empathy at private hospitals. This shows the 
importance of noting that patients' perception on the health care providers empathy can also be influenced by their personal characteristics.

Generally, four of the identified determinants were common for both categories of hospitals, public and private. But unique independent predictors of perceived provider empathyat private and public hospitals were also identified. Among these unique determinants of perceived provider empathy at public hospital, perceived cleanliness of hospital and perceived accessibility of the hospital services were included. Likewise, at private hospitals, open posture while taking with the patients and consultation duration were important determinants of perceived provider empathy.

Finally, we would like to remind the reader about the possibility of social desirability in this study as the respondents were interviewed in the compound of the health facility. It should also be noted that the reliance on the response of parents/care givers for their children may introduce surrogate bias.

\section{Conclusion}

In conclusion, the difference in the mean level of empathy at public and private hospitals was statistically significant. Despite this,four of the independent predictors of perceived provider empathy for respondents of both categories were common. The predictors of perceived provider empathy in this study were related to characteristics of patients, health care providers and the health system. Therefore, health managers and health care providers should work on improving the communication skills of health professionals which could significantly alter the perception of the patient to services $s /$ he receives at the outpatient department. Researchers are advised to further explore the nature of patient-provider interaction in resource limited settings such as the one this study was carried out.

\section{Authors' Contributions}

TT was involved in the conception, design, analysis, interpretation, report writing and manuscript writing. MW and SO had been involved in the design, analysis, interpretation and report writing. MW was also involved in manuscript writing. All authors read and approved the final manuscript.

\section{Acknowledgements}

We are highly indebted for all participants of the study, supervisors of data collection and data collectors for their worthy effort and participation in this study. We are also thankful to Jimma University for funding this study.

\section{References}

[1] Decety, J., Moriguchi, Y. The empathic brain and its dysfunction in psychiatric populations: implications for intervention across different clinical conditions. BioPsychoSocialMedicine. 2007; 1: 22.

[2] McKinnon, M.C., Levine, B., Moscovitch, M. Domaingeneral contributions to social reasoning: the perspective from cognitive neuroscience. In: Roberts, M.J. (Ed.), Integrating the Mind. Psychology Press, Hove, UK. 2007; $153-177$.

[3] Hojat M, Mangione S, Nasca TJ, Cohen MJM, Gonnella JS, Erdmann JB, Veloski JJ, Magee M. The Jefferson Scale of Empathy: development and preliminary psychometric data. Educational and Psycho Measurement. 2001; 61:349-365

[4] Aring CD. Sympathy and empathy. JAMA. 1958; 167:448452

[5] Registered Nurses Association of Ontario: Nursing Best Practice Guideline: Establishing therapeutic relationships. 2002

[6] Washington State Department of Health: Cultural Competency in Health Services and Care: A Guide for Health Care Providers. 2010

[7] James T Hardee: An Overview of Empathy.The Permanente Journal 2003; 7:4

[8] Mercer SW, Reynolds WJ. Empathy and quality of care. $\mathrm{Br}$ J Gen Pract. 2002 Oct;52 Suppl:S9-12.

[9] Suchman AL, Roter D, Green M, Lipkin M Jr. Physician satisfaction with primary care office visits. Collaborative Study Group of the American Academy on Physician and Patient. Med Care. 1993 Dec; 31(12):1083- 92.

[10] Kim S S, Kaplowitz S, Johnston M V. The effects of physician empathy on patient satisfaction and compliance. Eval Health Prof. 2004 Sep; 27(3):237-51.

[11] Vermeire E, Hearnshaw H, Van Royen P, Denekens J. Patient adherence to treatment: three decades of research. A comprehensive review. J Clin Pharm Ther. 2001 Oct; 26(5):331-42.

[12] Neumann M Edelhäuser F, Tauschel D, Fischer R. M, Wirtz M, Woopen C, Haramati A, Scheffer C. Empathy Decline and Its Reasons: A Systematic Review of Studies With Medical Students and Residents.Acad Med. 2011; 86: 9961009

[13] Rakel D, Barrett B, Zhang Z, Hoeft T, Chewning B, Marchand L, and Scheder J. Perception of Empathy in the Therapeutic Encounter: Effects on the Common Cold. Patient EducCouns. 2011; 85(3): 390-397

[14] Irving P, Dickson D. Empathy: towards a conceptual framework for health professionals. International Journal of Health Care Quality Assurance 2004; $17 \quad 4$ :212-220

[15] HojatM ,Gonnella J S, Nasca T J, et al. Physician Empathy: Definition, Components, Measurement, and Relationship to Gender and Specialty. Am J Psychiatry. 2002; 159:1563-69

[16] Machida J: Can empathy help healthcare and business? Qn: a publication of YalaSchool of management. 2008 
[17] Birhanu Z, AssefaT, Woldie M, Morankar S. Predictors of perceived empathy among patients visiting primary healthcare centers in central Ethiopia. International Journal for quality in health care. 2012

[18] Al Onazi M, Al Jondeby M, Azeem M, Al Sayyari A. Factors Affecting Saudi Hemodialysis Patients' Perception of Healthcare Providers' Empathy. Arab Journal of Nephrology and Transplantation. 2011; 4(2):71-6

[19] Hojat M, Louis D, Maxwell K, Markham F, Wender R, Gonnella J: Patient perceptions of physician empathy, satisfaction with physician, interpersonal trust, and compliance. Int. J. Medical Education. 2010; 1:83-87

[20] Birhanu Z, Woldie M K, Assefa T, Morankar S. Determinants of patient enablement at primary health care centers in central Ethiopia: a cross-sectional study. Afr J Prm Health Care Fam Med. 2011; 3(1)

[21] Mercer et al. Patient enablement requires physician empathy: a cross-sectional study of general practice consultations in areas of high and low socioeconomic deprivation in Scotland. BMC Family Practice. 2012 13:6.

[22] MacPherson H, Mercer SW, Scullion T et al. Empathy, enablement, and outcome: an exploratory study on acupuncture patients' perceptions. JAltern Complement Med.2003;9:869-76
[23] Neumann, M., Bensing, J., Wirtz, M., Wubker, A., Scheffer, C., Tauschel, D., Edelhauser, F., Ernstmann, N., Pfaff, H. The impact of financial incentives on physician empathy: a study from the perspective of patients with private and statutory health insurance. Patient Education and Counseling. 2011, 84(2), 208-216

[24] Schirmer JM, Mauksch L, Marvel MK et al. Assessing communication competence: a review of current tools. Fam Med. 2005;37:184-92

[25] Tateke T, Woldie M, Ololo S. Determinants of patient satisfaction with outpatient health services at public and private hospitals in Addis Ababa, Ethiopia. Afr J Prm Health Care Fam Med. 2012; 4(1)

[26] Hugh M, Stewart W, Tony S, Kate J. Empathy, Enablement, and Outcome: An Exploratory Study on Acupuncture Patients' Perceptions. The Journal of Alternative and Complementary Medicine. 2003; 9 (6) 869-876. http://www.liebertonline.com/doi/abs/10.1089/10755530377 1952226

[27] Hisholm A. Askham J. What do you think of your doctor? A review of questionnaires for gathering patients' feedback on their doctor. Picker institute, Europe; 2006. http://www.engage.hscni.net/library/What $\% 20 \mathrm{do} \% 20$ you $\% 2$ 0think.pdf 